Article

\title{
Optically Monitored Electric-Field-Induced Phase Transition in Vanadium Dioxide Crystal Film
}

\author{
Peng-Fei Wang ${ }^{1,+}$, Qianqian $\mathrm{Hu}^{2,+}$, Tan Zheng ${ }^{3,+}, \mathrm{Yu} \mathrm{Liu}^{1}$, Xiaofeng $\mathrm{Xu}^{3, *}$ and Jia-Lin Sun ${ }^{2, *}$ \\ 1 College of Mechanical Engineering and Automation, Fuzhou University, Fuzhou 350108, China; \\ wangpf@fzu.edu.cn (P.-F.W.); Liuyu19@fzu.edu.cn (Y.L.) \\ 2 Collaborative Innovation Center of Quantum Matter, State Key Laboratory of Low-Dimensional Quantum \\ Physics, Department of Physics, Tsinghua University, Beijing 100084, China; hqq16@mails.tsinghua.edu.cn \\ 3 Department of Applied Physics, Donghua University. No. 2999 North Renmin Road, Songjiang District, \\ Shanghai 201620, China; 2181620@mail.dhu.edu.cn \\ * Correspondence: xxf@dhu.edu.cn (X.X.); jlsun@tsinghua.edu.cn (J.-L.S.) \\ + These authors contributed equally to this work.
}

Received: 20 April 2020; Accepted: 28 August 2020; Published: 29 August 2020

\begin{abstract}
Vanadium dioxide $\left(\mathrm{VO}_{2}\right)$, due to its electrically induced metal-to-insulator transition with dramatic changes in electrical and optical properties, is considered to be a powerful material for electro-optical devices. However, there are still some controversies about phase transition mechanism under voltage. Here, based on optical characterizations on $\mathrm{VO}_{2}$ crystal nanofilm during the whole process of phase transition, temporal evolution and spatial distribution of changes in electricity, optic and temperature are investigated simultaneously, to explore the mechanism. The variations of Raman spectrum and reflected spectrum, and changes in current and temperature are evidences for occurrence of phase transition, which exhibit different changing behaviors with time and space. These results offer a better understanding of the phase transition mechanism, implying that lattice structure of $\mathrm{VO}_{2}$ changes gradually after applying voltage until the structure is completely converted to metallic structure, which causes a rapid increase in carrier density, resulting in a rapid change in current, reflected spectrum and temperature. Temperature rise before phase transition and applied electric field alone are not enough for triggering metal-insulator transition, but these two factors can act synergistically on structural transformation to induce phase transition.
\end{abstract}

Keywords: vanadium dioxide; phase transition; Raman spectrum; reflected spectrum; temperature; temporal evolution; spatial distribution

\section{Introduction}

For a long time, phase transition, especially metal-insulator transition (MIT), has been an important research topic for many researchers [1]. As a classic correlated oxide, vanadium dioxide $\left(\mathrm{VO}_{2}\right)$ undergoes a first-order phase transition from insulator to metal at near room temperature, accompanied by a structural change from the monoclinic structure of insulator to the rutile structure of metal [2,3], and has attracted long-standing interest in the condensed matter materials sciences. The significant change in the resistivity and long-wave transmittance [4-6] makes it have great potential in the electronic and optical field [7]. Nowadays, it has been applied to thermal sensors [8], chemical sensors [9], electrical oscillators [10], electronic switches [11,12], memristive device [13], smart windows [14] and so on. There are many kinds of external stimuli that can drive phase transition of $\mathrm{VO}_{2}$. Besides temperature, the MIT of $\mathrm{VO}_{2}$ can also be triggered by voltage [15-17], strain [18] and ultrafast laser excitation [19]. The trigger of phase transition by voltage is very important for the benefit of convenient integration with modern technology and large-scale application. However, the phase transition triggered by 
voltage is closely associated with heat generation [20], which leads to some controversial understanding of its internal physical mechanism, and limits its further development.

The exploration on the physical mechanism of phase transition has been a challenging subject, and sometimes poses controversial theoretical, as well as experimental problems. The focus of the debate is whether phase transition under voltage comes from electric-field mechanism involving injection of charge carriers from electrode or thermal switching mechanism involving inhomogeneous Joule heating, both of which have supportive theoretical or experimental results. The results that support the electronic mechanism as reason for phase transition are as follows: (1) it is experimentally determined that the time of phase transition under the effect of voltage can be on the order of nanoseconds or picoseconds, and it is generally believed that the Joule heat effects does not have such a fast speed [17,21,22]; (2) some modeling results point out that the heat generated by high-resistance $\mathrm{VO}_{2}$ under voltage is relatively small, just resulting in a small increase in temperature that is not enough to reach phase-transition temperature [23]. The proof that the effect of Joule heating cannot be ignored shows that the temperature of $\mathrm{VO}_{2}$ film under voltage, with the help of Poole-Frenkel mechanism and self-accelerating heating mechanism, can be raised to the required temperature for phase transition in nanosecond or picosecond $[20,24,25]$. The theories of Mott phase transition and Peierls phase transition are attempting to elucidate the mechanism, but cannot completely solve all phase-transition problems [26]. There are a few difficulties that arise when trying to determine whether thermal or electronic process are primarily responsible for voltage-induced conductivity switching in $\mathrm{VO}_{2}$ films. The main difficulties include a simple visualization of the phase transition region to determine location and volume of the region and direct synchronous measurement of important parameters during phase-transition process, such as temperature, current and transmittance at sufficient spatial and temporal resolution [20].

In this study, a small-size device based on $\mathrm{VO}_{2}$ crystal nanofilm are well prepared to observe and explore phase transition. Optical methods, such as microscopic spectroscopy imaging and infrared thermography, are adopted to characterize the changes of $\mathrm{VO}_{2}$ nanofilm in Raman spectrum, reflected spectrum and temperature from the beginning moment, when voltage is applied on the nanofilm, to the occurrence of phase transition of the nanofilm, and finally to the return of the film to its initial state after voltage is removed. The location and area of phase-transition region are exactly determined through simple microscopic imaging ways and phase-transition region can be simply modulated by changing external resistor and voltage. The characterizations of the phase-transition region and non-phase-transition region, are successfully carried out from the perspective of temporal evolution and spatial distribution. The collaborative analysis of the characterization results, in terms of time and space, and the comparative discussion of characterization results at different regions, provide more experimental proofs to help us reveal the phase transition of $\mathrm{VO}_{2}$ under voltage.

\section{Materials and Methods}

\subsection{Fabrication of Vanadium Nanofilm}

The film is grown on c-cut sapphire substrate by a simple method using sputtering then oxidation in air. A vacuum chamber is filled with working gas $\operatorname{Ar}$ (99.99\% purity, Beijing, China). Under the conditions of $1.2 \times 10^{-1} \mathrm{~Pa}$ and $136 \mathrm{~W}$, metal vanadium nanofilm (99.99\% purity, Shanghai, China) is deposited on the substrate through DC sputtering. The distance between the target and substrate is fixed at $120 \mathrm{~mm}$. The substrate is rotated at $13 \mathrm{rpm}$ to ensure uniformity of thin film deposition. In order to eliminate contamination on the target surface, pre-sputtering of the vanadium metal target is performed for $10 \mathrm{~min}$, before depositing the metallic vanadium nanofilms. The thicknesses of vanadium thin films can be controlled with deposition time. 


\subsection{Fabrication of $\mathrm{VO}_{2}$ Nanofilm and Electrodes}

Vanadium nanofilm is put into the annealing furnace for further oxidation at the temperature of $470{ }^{\circ} \mathrm{C}$. The oxidation is carried out in air conditions. To control the oxidation process, the Ar gas ( $99.99 \%$ purity, Beijing, China) is filled into the annealing furnace at a speed of $70 \mathrm{~mL} / \mathrm{min}$. Then two uniform gold thin films (99.9\% purity, Beijing, China) with spacing of $75 \mu \mathrm{m}$, width of $110 \mu \mathrm{m}$ and thickness of $35 \mathrm{~nm}$, are evaporated on $\mathrm{VO}_{2}$ nanofilm as electrodes under the vacuum of $10^{-3} \mathrm{~Pa}$. For the measurement of temperature, a device with electrode spacing of $3 \mathrm{~mm}$ and width of $10 \mathrm{~mm}$ is also prepared using the same method.

\subsection{Characterization}

Electric measurements of $\mathrm{VO}_{2}$ device are measured by Source-Meter (Keithley 2400, Shanghai, China). Optical micrograph images, Raman spectrums and reflected spectrums are carried out with multifunctional and high resolution near-field optoelectronic and spectroscopic imaging microscope (Alpha300RAS, WITec, Beijing, China). The linearly polarized 532-nm laser is used as light source for the Raman spectrum. The diameter of laser spot is $300 \mathrm{~nm}$ and the adopted laser power is $5 \mathrm{~mW}$. A halogen lamp is used as light source to measure reflected spectrum. The beam of white light emitted from Halogen lamp has a diameter of about $1 \mu \mathrm{m}$ and power of about $10 \mathrm{~mW}$. The infrared thermography and temperature measurements are carried out with dedicated type thermal imager (GF706, DALI Technology, Hangzhou, China).

\section{Results and Discussion}

In order to observe and investigate the phase transition process and the mechanism of $\mathrm{VO}_{2}$ nanofilm under external voltage by utilizing optical methods in detail, a $\mathrm{VO}_{2}$ nanofilm on the sapphire substrate is prepared as described in Materials and Methods section. Although vanadium has many oxides with different ratios of vanadium and oxygen, the production of other oxides can be reduced in the preparation process by controlling the oxygen conditions, resulting in relatively pure $\mathrm{VO}_{2}$ nanofilm. The 62-nm-thick $\mathrm{VO}_{2}$ nanofilm is composed of grains with an average diameter of $55.4 \mathrm{~nm}$, with a root-mean square (RMS) surface roughness of about $6.97 \mathrm{~nm}$ and a high crystal orientation (020). The other detailed characterization on the prepared $\mathrm{VO}_{2}$ nanofilm can be seen in our previous work [27], and the results tell us that the $\mathrm{VO}_{2}$ nanofilm is well prepared without obvious defects. Figure 1a,b show schematic diagram of measuring device and optical microscopic image of real sample respectively. The changes in electric parameters of $\mathrm{VO}_{2}$ nanofilm such as flowing current and resistance, are measured since voltage is applied. From multiple experiments, we found that high voltage can damage nanofilm after phase transition of $\mathrm{VO}_{2}$ nanofilm occurs, so an external resistor is necessary to connect with nanofilm in series, for the protection of $\mathrm{VO}_{2}$ nanofilm and the acquisition of phase-change data. The value of external resistor depends on applied voltage and resistance of $\mathrm{VO}_{2}$ nanofilm. As can be seen from Figure 1c, temperature drives the MIT of prepared $\mathrm{VO}_{2}$ sample at approximately $56^{\circ} \mathrm{C}$, and the temperature range required by phase transition is about $5 \mathrm{~K}$, which is consistent with reported results $[26,28,29]$. The temperature point where phase transition occurs can be influenced by many factors, such as doping, stoichiometry and strain, so acceptable differences in phase-transition temperature exist for different samples. After phase transition, the resistance of $\mathrm{VO}_{2}$ film changes from $203 \mathrm{~K} \Omega$ at low temperature to $25 \Omega$ at high temperature, with a change of about four orders in magnitude. Figure $1 \mathrm{~d}$ exhibits the phase transition of $\mathrm{VO}_{2}$ nanofilm under voltage when external resistor is $0 \Omega$. As voltage increases from $0 \mathrm{~V}$, flowing current increases slowly with voltage, and then an abrupt jump of current occurs at about $38 \mathrm{~V}$, where the corresponding electric filed is about $10^{5} \mathrm{~V} / \mathrm{m}$ (electrode spacing is $75 \mu \mathrm{m}$, indicating that the film experiences phase transition and turns into metal phase. For the subsequent decreasing process of voltage, the metal phase can remain until the voltage decreases to $4 \mathrm{~V}$. The similarity of the phase-transition curve on the positive and negative sides of the coordinate axis proves the symmetrical device structure and good film quality. 


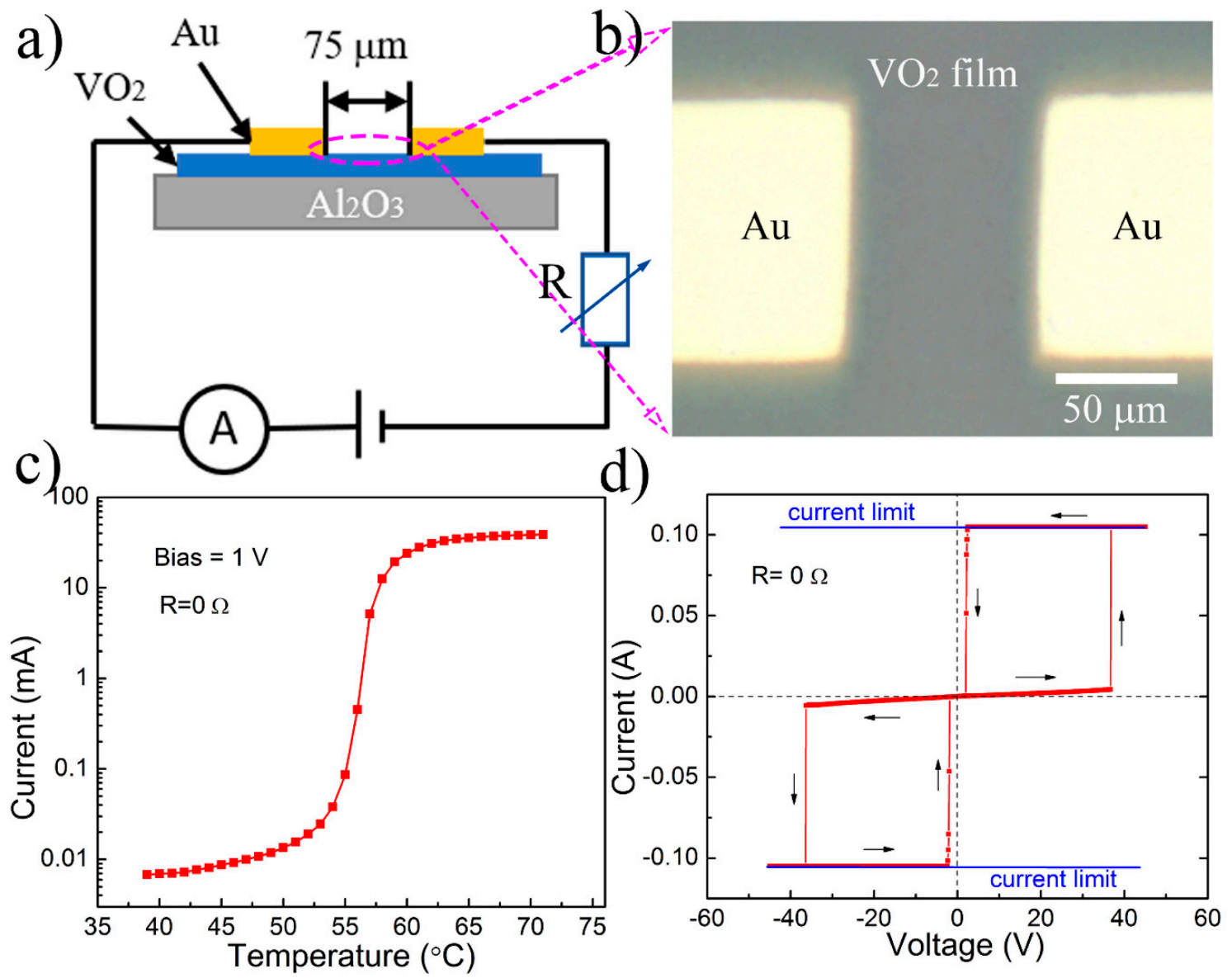

Figure 1. The preparation of vanadium dioxide $\left(\mathrm{VO}_{2}\right)$ device and phase-transition characterization under the effect of temperature and voltage. (a) Schematic diagram of device for the measurement of phase transition. $\mathrm{R}$ is external resistor. (b) Optical microscope image of the prepared $\mathrm{VO}_{2}$ sample. The Au nanofilm as electrodes is deposited on $\mathrm{VO}_{2}$ nanofilm. (c) The current of $\mathrm{VO}_{2}$ film versus temperature without external resistor at a voltage of $1 \mathrm{~V}$. (d) The evolution of current versus voltage at room temperature without external resistor.

For the measurements shown in Figure 2, the external resistor is $130 \mathrm{~K} \Omega$, and a source-drain voltage of $400 \mathrm{~V}$ is applied in the circuit to trigger phase transition. As shown in the Figure $2 \mathrm{a}, \mathrm{VO}_{2}$ nanofilm undergoes phase transition after an incubation time of about $80 \mathrm{~s}$ since voltage is applied. The incubation time would become shorter with the increase of voltage, as shown in Figure S1 in Supplementary Materials. An optical microscope is used to observe the real-time image of $\mathrm{VO}_{2}$ nanofilm after application of voltage. When flowing current of nanofilm jumps, a long blue region with a width of $1.9 \mu \mathrm{m}$ appears on the film, connecting the source and drain electrodes (Figure 2b). The emerging blue region remains stable and does not change with the duration of voltage. If the external resistor does not change, the position and shape of the blue region will not change, even if the voltage is reapplied after being removed. The width of the blue region can be modulated by changing the external resistor and source-drain voltage as shown in Figure S2. We select position "O" (shown in Figure $2 \mathrm{~b}$ ) in the blue region as observation point for following measurements. A beam of white light (Halogen lamp as light source) with a diameter of about $1 \mu \mathrm{m}$ is illuminated on the position " $\mathrm{O}$ ", and the reflected spectrum of visible light from $430 \mathrm{~nm}$ to $700 \mathrm{~nm}$ is collected (Figure 2c). It can be found that the intensity of reflected short-wavelength light stays unchanged and the intensity of reflected long-wavelength light decreases after phase transition. That is also why this region becomes blue, as observed in Figure 2b. Further, we observe the change of reflected light intensity during the process of applying/removing of voltage (Figure 2d). The evolution of the reflected light intensity with time has 
a sudden drop when the phase transition occurs and a sudden recovery when voltage is removed, which is consistent with the change of current in Figure 2a.

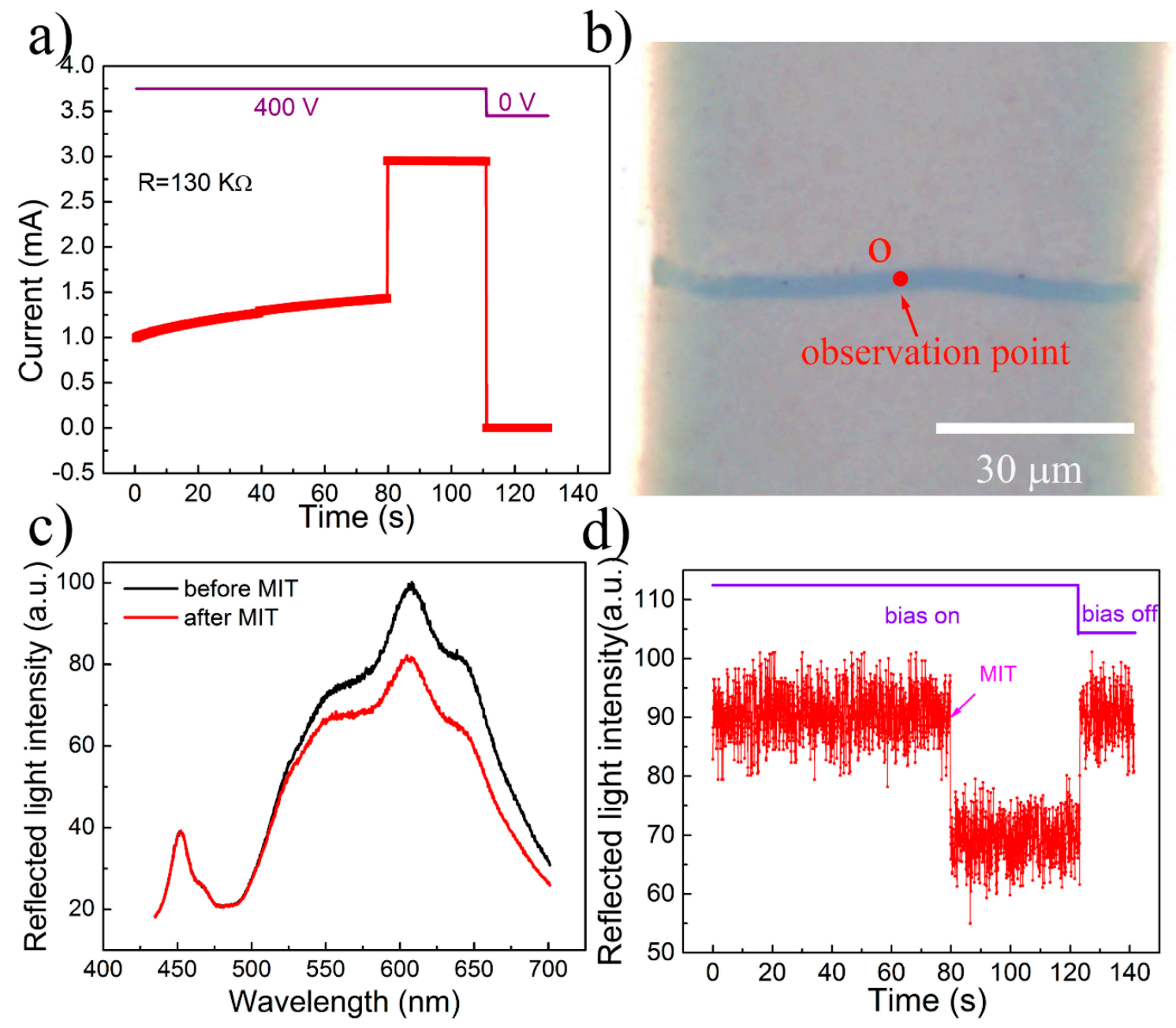

Figure 2. A voltage of $400 \mathrm{~V}$ is applied on the circuit with a $130 \mathrm{~K} \Omega$ external resistor. (a) The evolution of flowing current with time. (b) Optical microscope image of $\mathrm{VO}_{2}$ film after phase transition. The red dot "O" is observation point of the following tests. (c) Reflected spectrum before and after phase transition. The light source is white light emitted from halogen lamp. (d) The evolution of reflected light intensity from $430 \mathrm{~nm}$ to $700 \mathrm{~nm}$ over time when applying voltage and withdrawing voltage.

Next, we observe the evolution of Raman spectrum with time at point " $\mathrm{O}$ " when voltage is applied or removed on the $\mathrm{VO}_{2}$ nanofilm (Figure 3). Like reflected spectrum, Raman spectrums are measured at the same device and the same observation point under the same voltage. When no voltage is applied, the nanofilm is in the insulating phase and the Raman spectrum is the black line shown in the Figure 3a. The black line has seven distinct peaks, including 193, 223, 309, 340, 392, 499 and $613 \mathrm{~cm}^{-1}$ peaks, which are consistent with the previous report [30]. After applying voltage, the intensities of all the peaks gradually decrease with the increase of time, but the peak positions and widths do not have an obvious change. This is an evidence that transition is going on [31]. When phase transition occurs, all the seven peaks finally disappear, and a broad peak is obviously observed at $530 \mathrm{~cm}^{-1}$, which is a characteristic peak of metallic $\mathrm{VO}_{2}$ [30]. We select the areas of $613 \mathrm{~cm}^{-1}$ peak (representing insulating phase of $\mathrm{VO}_{2}$ ) and $530 \mathrm{~cm}^{-1}$ peak (representing metallic phase of $\mathrm{VO}_{2}$ ) as variables, and monitor their changes during the process of applying and removing the voltage (Figure $3 \mathrm{~b}$ ). It can be found that the area of $613 \mathrm{~cm}^{-1}$ peak continuously decreases after the voltage is applied. The zero intensity is observed at the time of phase transition and remains unchanged afterwards. Correspondingly, the 
area of the $530 \mathrm{~cm}^{-1}$ peak continuously increases from zero to the maximum value and then remains stable. The evolution process of Raman peaks indicates that $\mathrm{VO}_{2}$ nanofilm experiences changes from the beginning of applying voltage. After the voltage is removed, the Raman spectrum gradually return to the initial state. Compared with recovery of reflected light in Figure $2 \mathrm{~d}$, more time is needed for recovery of Raman spectrum, due to the high temperature formed by high voltage and low resistance of metallic $\mathrm{VO}_{2}$. The recovery process is dominated by a slow dissipation of heat to external environment. The changes in Raman spectrum can be cycled with the applying and removing of voltage.

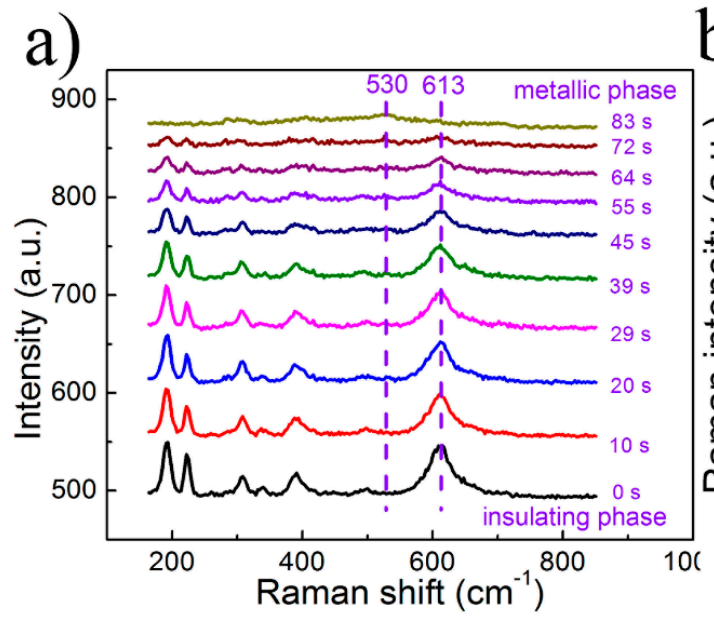

b)

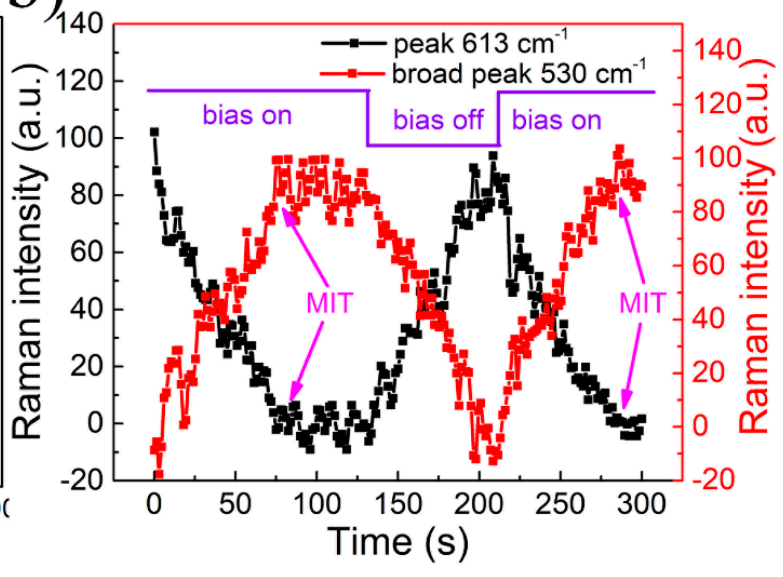

Figure 3. The evolution of Raman spectrum with time under the effect of voltage at a voltage of $400 \mathrm{~V}$ with a $130 \mathrm{~K} \Omega$ external resistor in the circuit. (a) Raman spectrum of $\mathrm{VO}_{2}$ nanofilm at different times during its transition from insulating phase to metallic phase. (b) The change of peak areas of $613 \mathrm{~cm}^{-1}$ and $530 \mathrm{~cm}^{-1}$ versus time after applying or removing voltage.

From the above results, we know that the blue region appears with the jump of current, and it possesses the changes in the reflected spectrum and the Raman spectrum, proving that the blue region is the phase-transition region. The reflected spectrum and the Raman spectrum of this region have different changing behavior after application of voltage, especially gradual change of the Raman spectrum and sudden change of reflected spectrum. The observed gradual transition of the Raman spectrum indicates that the $\mathrm{VO}_{2}$ nanofilm starts to undergo some changes as soon as the electric field is applied, which is an interesting phenomenon to be explored. On the one hand, through the previous characterizations, we know that the prepared $\mathrm{VO}_{2}$ nanofilm is composed of crystal grains with the similar orientation. As proved in reported work [32], the phase transition of the whole $\mathrm{VO}_{2}$ nanofilm is made up of stepwise phase transition of different crystal grains. For $\mathrm{VO}_{2}$ nanofilm under voltage, a stepwise phase transition of grains is much faster than the change of Raman spectrum shown in Figure $3 b$ [32], thus, the process of grains' stepwise phase transition is not the reason for the gradual change of the Raman spectrum. On the other hand, we know that the Raman spectrum reflects the information of lattice structure through detecting lattice vibration modes, so the change of the $\mathrm{VO}_{2}$ nanofilm revealed by the Raman spectrum should be about structure [4]. Meanwhile, the $530 \mathrm{~cm}^{-1}$ peak as a symbol of metallic phase of $\mathrm{VO}_{2}$, increases as soon as the electric field is applied [33,34], and further supports the judgement about structural change. When the voltage is applied to the $\mathrm{VO}_{2}$ nanofilm, some ions and electrons in the lattice of $\mathrm{VO}_{2}$ move, resulting in the slow transition of the monoclinic structure of $\mathrm{VO}_{2}$ in the insulating phase. The crystal structures of $\mathrm{VO}_{2}$ within incubation time are intermediate states. The deviation of intermediate state from the insulating monoclinic state or metal rutile state determines the intensities of the corresponding Raman spectrums. The gradual change of lattice structure is companied with gradual increase of flowing current, as shown in the Figure 2a, suggesting that electron conductivity is also gradually increasing. The bandgap of initial insulating phase changes with the transition of lattice structure. When the lattice structure of $\mathrm{VO}_{2}$ 
finally turns into rutile structure, the carrier density has a sudden increase due to disappear of bandgap, resulting in sudden increase in electrical conductivity. The reflected spectrum is associated with lattice structure and carrier density, and different electronic states present a difference in optical color [26]. Thus, a change in reflected spectrum would occur when carrier density has obvious difference, as shown in Figure $2 \mathrm{c}, \mathrm{d}$. When voltage is removed, the metal phase of the $\mathrm{VO}_{2}$ nanofilm disappears, so carrier density decreases, resulting in a sudden change of electrical conductivity and the reflected spectrum. The accurate measurement of lattice change under voltage is very difficult. The great challenge for accurate characterization on $\mathrm{VO}_{2}$ lattice structure comes from the limitation of currently available characterizations, in that it is indeed hard to capture intermediate electronic states in spatial isolation because of their transient occurrence [26].

Further, we characterized the spatial distribution of the Raman spectrum and reflected spectrum on the $\mathrm{VO}_{2}$ nanofilm after phase transition occurs (Figure 4). Similarly, with an external resistor of $130 \mathrm{~K} \Omega$, a voltage of $400 \mathrm{~V}$ results in phase transition of $\mathrm{VO}_{2}$ nanofilm and blue region appears on the film (Figure 4a). The blue box shown in Figure 4a are measuring area where the Raman spectrum and reflected spectrum are respectively collected through a way of point-by-point scanning. The detailed Raman spectrums at different positions can be seen in Figure S3. Figure $4 \mathrm{~b}$ shows the mapping of the Raman peak area of $613 \mathrm{~cm}^{-1}$. Figure 4c shows the mapping of intensity of reflected light from $430 \mathrm{~nm}$ to $700 \mathrm{~nm}$. To better observe the changes of two spectrums in space, the distribution of peak areas of $613 \mathrm{~cm}^{-1}$ and $530 \mathrm{~cm}^{-1}$ (Figure 4d), and the reflected light intensity (Figure 4e), along the red line in Figure $4 \mathrm{a}$, are respectively exhibited. The width of the blue region in Figure $4 \mathrm{a}$ is about $1.9 \mu \mathrm{m}$, and the width of the black band in Figure $4 \mathrm{c}$ is also about $1.9 \mu \mathrm{m}$. From Figure $4 \mathrm{e}$, we can obtain that the curve of reflected light intensity drops near the position coordinate of $30 \mu \mathrm{m}$, and the full width at half height of the peak is also about $1.9 \mu \mathrm{m}$. These results consistently show that only blue region with a width of about $1.9 \mu \mathrm{m}$ have a transition from an insulating phase to a metallic phase. Therefore, phase-transition region can be directly determined through the optical microscope. Raman spectral imaging of the $613 \mathrm{~cm}^{-1}$ peak in Figure $4 \mathrm{~b}$, and the intensity distributions of the $613 \mathrm{~cm}^{-1}$ peak and $530 \mathrm{~cm}^{-1}$ peak in Figure $4 \mathrm{~d}$ along with the red line, show that the lattice structure of $\mathrm{VO}_{2}$ is distorted in space under the action of voltage within the region, with a width of about $50 \mu \mathrm{m}$, centered at the position coordinate of $30 \mu \mathrm{m}$. The area of the $613 \mathrm{~cm}^{-1}$ peak is zero within the blue region. Both sides of blue region are accompanied with regions where peak area of $613 \mathrm{~cm}^{-1}$ gradually increases with the increase of distance from blue region; correspondingly, the peak area of $530 \mathrm{~cm}^{-1}$ gradually decreases from the maximum value. We call the region with above changes the slowly-changing region of Raman. Within the slowly-changing region, on the one hand, the unchanging reflected spectrum indicates that it does not have the properties of metallic rutile phase; on the other hand, the gradual changing of areas of $530 \mathrm{~cm}^{-1}$ and $613 \mathrm{~cm}^{-1}$ peaks with distance tells us that slowly-changing region have changes in lattice structure. Further, the slowly changing region lies between phase-transition region and unaffected region, connecting the rutile structure and the monoclinic structure. It is reasonable that the structure in the slowly-changing region have a gradual transition in the lattice structure from rutile phase to monoclinic phase with the increase of distance from phase-transition region to achieve a smooth transition of the two structures in space.

There are many factors that can have an influence on the occurrence of phase transition. On the one hand, the long-time illumination of 532-nm laser used to measure the Raman spectrum and white light used to measure the reflected spectrum on the $\mathrm{VO}_{2}$ nanofilm, as shown in Figure S4, only caused a small increase in the current flowing through the film and did not trigger the phase transition, so the effect of illumination, such as heating, can be ignored. On the other hand, temperature and electric field both can act as triggers for phase transition. For $\mathrm{VO}_{2}$ nanofilm under voltage, temperature increase caused by Joule heating and high electric field caused by high voltage are both involved in the process of phase transition. More experiments are needed, and detailed discussion about the mechanism phase transition can be seen below. 

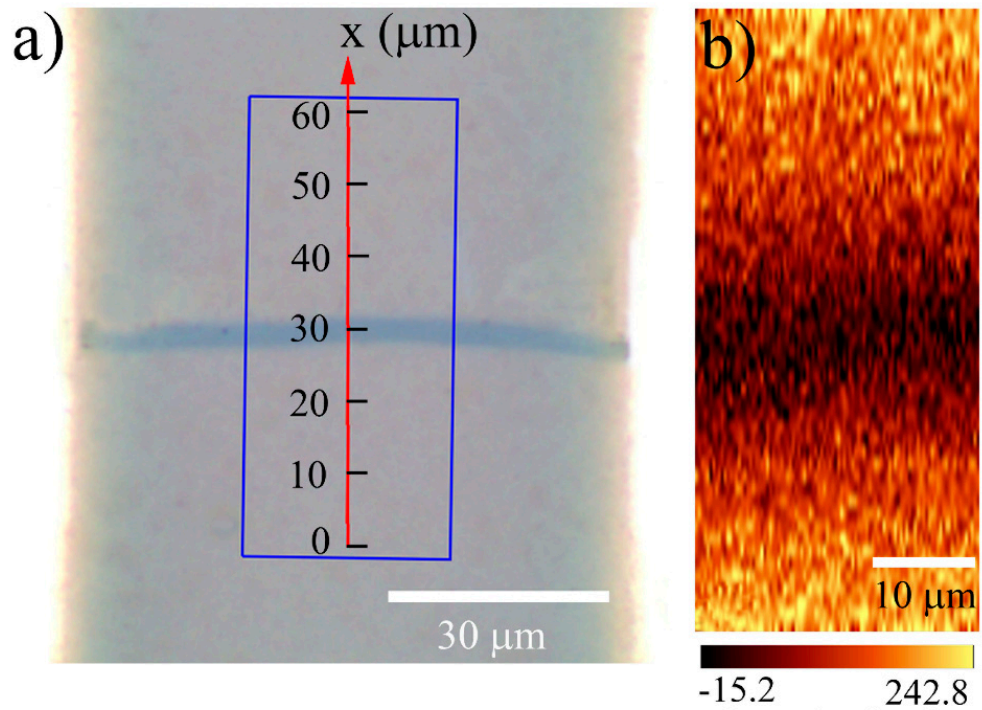

e)

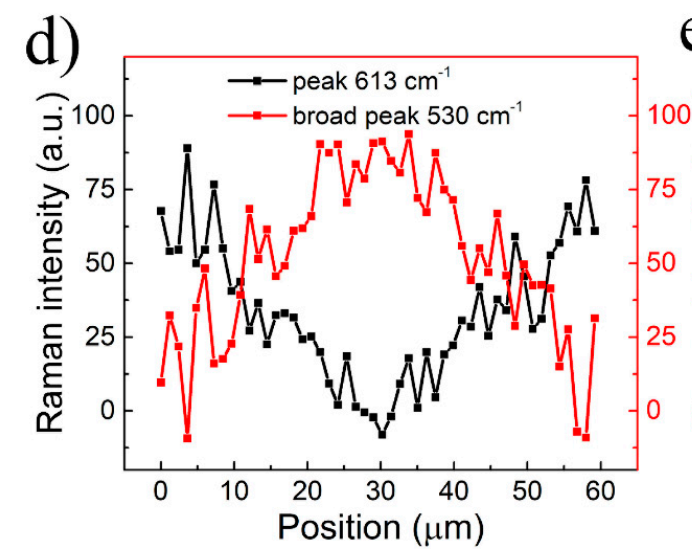

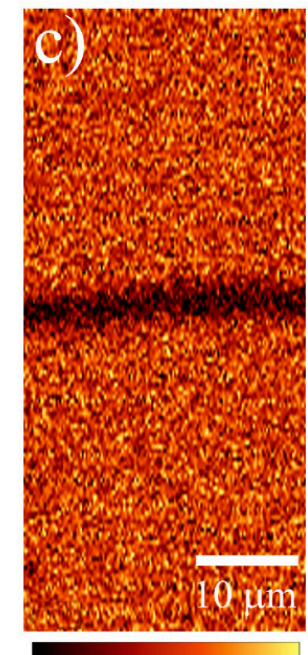

$-100$

6258

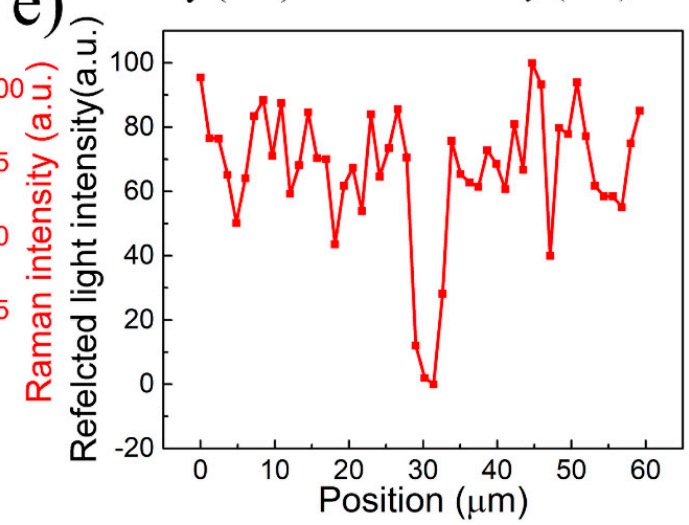

Figure 4. The spatial distribution of the Raman spectrum and the reflected spectrum at a voltage of $400 \mathrm{~V}$ with a $130 \mathrm{~K} \Omega$ external resistor in the circuit. (a) Optical microscope image of $\mathrm{VO}_{2}$ film after phase transition; $(\mathbf{b}, \mathbf{c})$ are mappings of peak area of $613 \mathrm{~cm}^{-1}$ and reflected light intensity from $530 \mathrm{~nm}$ to $700 \mathrm{~nm}$ in the box area shown in the Figure $4 \mathrm{a}$, respectively. (d) The change of peak areas of $613 \mathrm{~cm}^{-1}$ and $530 \mathrm{~cm}^{-1}$, and (e) the change of reflected light intensity along the red line in the Figure 4a.

In order to explore the driving mechanism of phase transition under voltage, the temperature of $\mathrm{VO}_{2}$ nanofilm is characterized. Limited by the spatial resolution of infrared camera (pixel size $100 \mu \mathrm{m}$ ), we expanded the electrode spacing to $3 \mathrm{~mm}$, which is about ten times bigger than before, to better obtain the change of temperature with time and space. All other conditions for the nanofilm remained unchanged. Unlike the obvious changes in defect concentration and scattering efficiency from the dimensional change of $\mathrm{VO}_{2}$ materials [26,35], the increase of electrode spacing mainly changes the resistance of $\mathrm{VO}_{2}$ nanofilm. The applied voltage becomes $800 \mathrm{~V}$, which is larger than before due to larger resistance. Figure 5a exhibit the temperature of nanofilm before phase transition. The temperature of the whole nanofilm between Au electrodes has a roughly uniform rise from room temperature to $37^{\circ} \mathrm{C}$ during the incubation time. After the phase transition occurs, as we can see in the Figure $5 \mathbf{b}$, blue phase-transition region appears, and the position of the phase-transition region is not located in the middle of the counter electrode, because the phase transition occurs preferentially in the path with the closest electrode spacing. The width of phase-transition region is $1.8 \mathrm{~mm}$, which is 100 times larger than pixel size of infrared camera, so sufficient data can be obtained to accurately characterize the temperature change. Comparing the test condition with the previous in Figure 2a, we can see that the electric field and flowing current density through $\mathrm{VO}_{2}$ nanofilm have both decreased, and, thus, the maximum value of incubation time of phase transition expands to $150 \mathrm{~s}$ (Figure $5 \mathrm{c}$ ). 
When applying an external electric field to observe the phase-transition behavior of the $\mathrm{VO}_{2}$ sample, the influence of the time interval between two adjacent measurements on its incubation time must be fully considered. Due to the expansion of the device size, two consecutive measurements need a longer interval time, otherwise, the residual temperature of the previous measurement will affect the incubation time of the next measurement. Figure $5 \mathrm{~d}$ shows that from the beginning of applying voltage, the temperature of the sample slowly and linearly rises to around $50{ }^{\circ} \mathrm{C}$ from room temperature, and then the phase transition suddenly occurs. In the region where point $\mathrm{A}$ is, the temperature of $\mathrm{VO}_{2}$ nanofilm after the phase transition rises rapidly to about $58^{\circ} \mathrm{C}$, due to the Joule heating caused by a sudden large current. Correspondingly, the temperature in non-phase-transition region (such as the region where point $\mathrm{B}$ is) has a small decrease. The decrease originates from the change in the flowing current. Although the total flowing current in $\mathrm{VO}_{2}$ nanofilm increases, the conductive path formed by phase-transition region accounts for most of it, and the flowing current in the non-phase-transition region becomes smaller compared to before. The temperature at sites $\mathrm{A}$ (phase-transition region) and $\mathrm{B}$ (non-phase-transition region) each stably stays $58^{\circ} \mathrm{C}$ and $45.3^{\circ} \mathrm{C}$, respectively. Figure $5 \mathrm{e}$ shows that the temperature symmetrically decreases on both sides of the phase transition region.
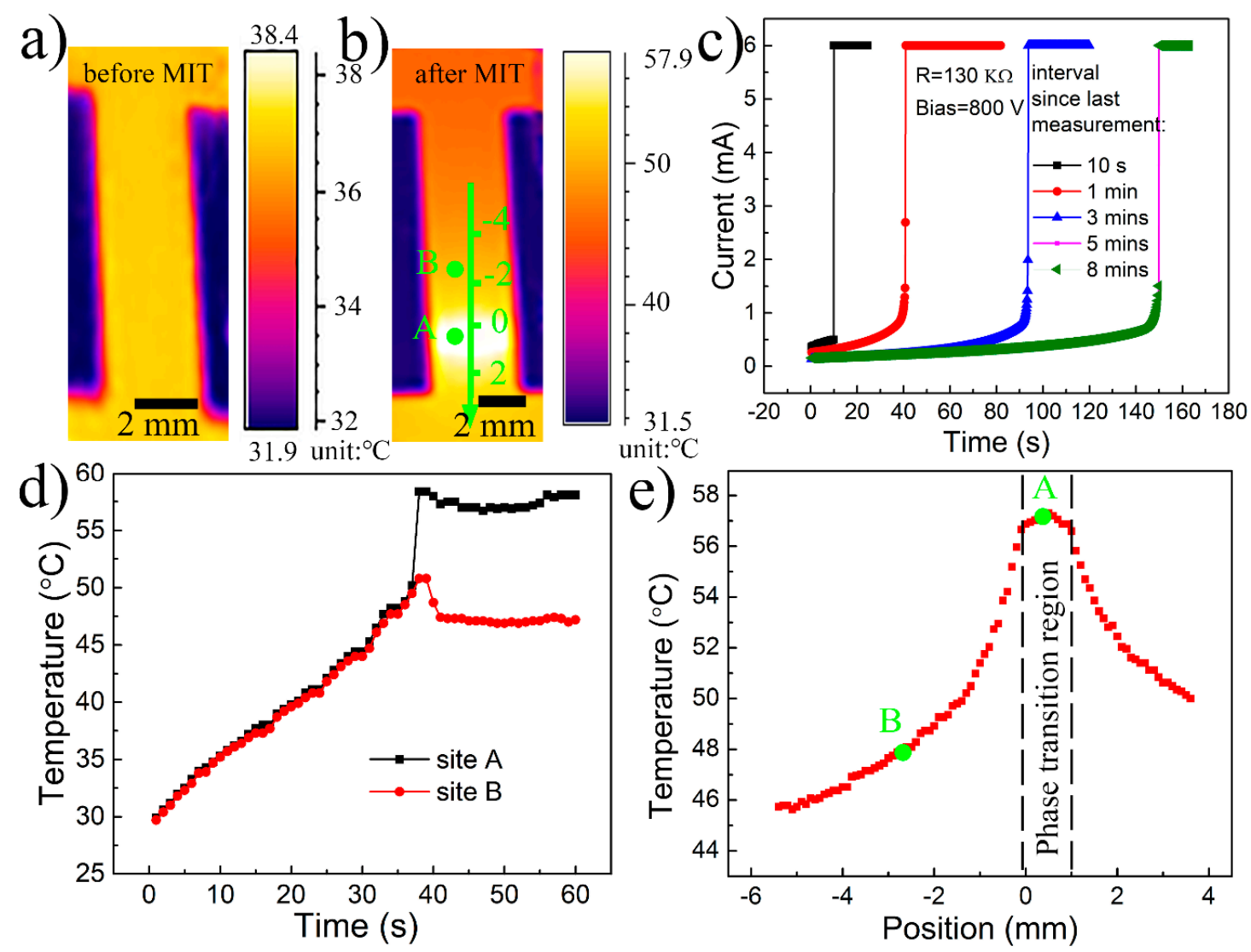

Figure 5. The temperature mapping images of $\mathrm{VO}_{2}$ nanofilm at a voltage of $800 \mathrm{~V}$ with a $130 \mathrm{~K} \Omega$ external resistor in the circuit (a) before and (b) after phase transition. Site A, B and the green line are observation positions for the following measurements. The length and width of electrodes are $10 \mathrm{~mm}$ and $3 \mathrm{~mm}$. (c) The evolution of flowing current with the incubation time after different time intervals since last measurement. The time intervals are $10 \mathrm{~s}, 1 \mathrm{~min}, 3 \mathrm{~min}, 5 \mathrm{~min}$ and $8 \mathrm{~min}$ respectively. The applied voltage is $800 \mathrm{~V}$ and the external resistor is $130 \mathrm{~K} \Omega$. (d) The temperature evolution of site A in the phase-transition region and site $\mathrm{B}$ in the slowly-changing region. The time interval since last measurement is $1 \mathrm{~min}$. (e) Temperature characterization along the line shown in the Figure 5b.

As shown in Figure $5 \mathrm{~d}$, for the $\mathrm{VO}_{2}$ nanofilm under a voltage of $800 \mathrm{~V}$, the temperature has a linear increase during the incubation time. The phenomenon that the temperature of $\mathrm{VO}_{2}$ sample in different 
areas shows a synchronous and linear increase, proves that only the Joule heating of flowing current is working for the temperature that increases to $48.2{ }^{\circ} \mathrm{C}$. The other mechanisms that can accelerate the increase of temperature is not obvious in this incubation time. The whole nanofilm, whether it's the area where point $A$ or point $B$ is, has the same temperature and keeps the temperature increasing synchronously before the phase transition, as shown in Figure S5. On the one hand, If the Joule heating mechanism causes the sample to undergo phase transition, the phase change at these two sites should happen at the same time. In fact, this is not the case. On the other hand, if phase transition of different grains in nanofilm starts to occur at $48.2{ }^{\circ} \mathrm{C}$ under voltage, Joule heating may dominate the process of phase transition. However, two observed experimental results do not support this proposal. First, the spatial distribution of temperature in Figure 5e indicates that the width of the region where the temperature is higher than $48.2^{\circ} \mathrm{C}$ would reach $7 \mathrm{~mm}$, which is 7 times wider than the observed width of phase-transition region in Figure $5 \mathrm{~b}$. Second, phase-transition region is accompanied with the change in reflected spectrum. The region with temperature changes from $48.2^{\circ} \mathrm{C}$ to $56{ }^{\circ} \mathrm{C}$ should have a gradual change in the number of phase-transition grains as reported work [32], which would result in the gradual change of reflected light intensity with space. However, this phenomenon is not observed in Figure 4c,e. Thus, the starting temperature of phase transition would not be $48.2^{\circ} \mathrm{C}$. The deceived results based on Joule heating mechanism are not consistent with the observed results, so Joule heating would not be the mechanism of phase transition. Then the attention is put on the electric field. In our device, on the one hand, the applied electric field is about $10^{5} \mathrm{~V} / \mathrm{m}$ (Figure 2) or less (Figure 5), which is less than the estimated critical electric field $(50 \mathrm{~V} / \mu \mathrm{m})$ required to induce MIT under the effect of electric field in three-terminal devices [31]. On the other hand, the incubation stage exists for the nanofilm under voltage in our two-terminal device. Generally, the structural phase transition and electronic phase transition of $\mathrm{VO}_{2}$ nanofilm occur nearly at the same time due to intense electron-lattice coupling [26]. Temperature and electric field both have been proven to be efficient ways for structural phase transition. After applying voltage on the film, electric field and Joule heating exist at the same time, and these two factors have a synergistic effect, which can reduce the requirements of individual factors on triggering phase transition. Therefore, we think that the two factors together induce phase transition. As voltage is applied, the lattice structure of $\mathrm{VO}_{2}$ film starts to change. The electric field drives the relative movement of some ions on lattice points of $\mathrm{VO}_{2}$. At the same time, electric field will drive charged carriers in the $\mathrm{VO}_{2}$ film to move, and the generated current will cause Joule heating, which will cause the local temperature of the film to rise. The expansion of vanadium oxide films caused by temperature rise is beneficial for further structural and electronic transition. The lattice structure parameter of the $\mathrm{VO}_{2}$ film changes slowly until it is completely in the rutile phase and the metal phase forms. As soon as phase transition occurs, the resistance rapidly decreases by several orders of magnitude, accompanying with the sudden change in reflectivity and temperature. When the voltage is removed, the metallic rutile phase disappears, and the current jumps to zero, resulting in the change of the reflectivity to its initial value. Without production of heat, the $\mathrm{VO}_{2}$ nanofilm dissipate heat slowly, so the lattice structure parameters will change slowly, and finally return to the initial state.

\section{Conclusions}

In summary, by means of the experimental characterizations of the Raman spectrum, the reflected spectrum and temperature on $\mathrm{VO}_{2}$ crystal nanofilm prepared by a simple method using sputtering and oxidation, we explored the process of phase transition under voltage. From the temporal evolution and spatial distribution of Raman spectrums, reflected spectrums and temperature, the process of phase transition under voltage is proved, which starts with gradual change in the lattice structure accompanying with changes in electrical, optical and thermal properties. The occurrence of the phase transition prompts the formation of the metal phase, leading to a sudden change in every aspect, including the reflected spectrum, current and temperature. The measurements of temperature and voltage show that the temperature increase before the phase transition and applied electric field are finite and not enough to drive the occurrence of phase transition alone, thus, indicating that two 
factors act synergistically on structural transformation to trigger phase change. As a controllable and convenient means, a better understanding of electrical control on the phase transition of $\mathrm{VO}_{2}$ nanofilm means it has great prospects in the application of ultrafast electronic devices.

Supplementary Materials: The following are available online at http://www.mdpi.com/2073-4352/10/9/764/s1, Figure S1: The relationship of incubation time and applied voltage, Figure S2: The relationship between current and width of phase-transition blue region, Figure S3: Raman spectrum of different positions on $\mathrm{VO}_{2}$ nanofilm, Figure S4: The influence of illumination of detecting light on the nanofilm, Figure S5: The temperature characterization of the nanofilm during the incubation time.

Author Contributions: P.-F.W. performed and analyzed the experimental measurements, and wrote the manuscript. Q.H. performed the supplementary experiments and analyzed the results. T.Z. performed and analyzed the growth of materials. Y.L. provided methods for the measurements. X.X. and J.-L.S. reviewed the manuscript and supervised the project. All authors have read and agreed to the published version of the manuscript.

Funding: This work was partially supported by NSAF (grant No. U1730246), the Research Fund Program of the State Key Laboratory of Low-Dimensional Quantum Physics (No. KF201704 and No. ZZ201703) and the Natural Science Foundation of Shanghai (Grant No. 16ZR1447800).

Conflicts of Interest: The authors declare no conflict of interest.

\section{References}

1. Yang, Z.; Ko, C.; Ramanathan, S. Oxide electronics utilizing ultrafast metal-insulator transitions. Annu. Rev. Mater. Res. 2011, 41, 337-367. [CrossRef]

2. Imada, M.; Fujimori, A.; Tokura, Y. Metal-insulator transitions. Rev. Mod. Phys. 1998, 70, 1039. [CrossRef]

3. Dernier, P.D.; Marezio, M. Crystal structure of the low-temperature antiferromagnetic phase of $\mathrm{V}_{2} \mathrm{O}_{3}$. Phys. Rev. B 1970, 2, 3771. [CrossRef]

4. Kucharczyk, D.; Niklewski, T. Accurate X-ray determination of the lattice parameters and the thermal expansion coefficients of $\mathrm{VO}_{2}$ near the transition temperature. J. Appl. Cryst. 1979, 12, 370-373. [CrossRef]

5. Jerominek, H.; Picard, F.; Vincent, D. Vanadium oxide films for optical switching and detection. Opt. Eng. 1993, 32, 2092-2100. [CrossRef]

6. Chain, E.E. Optical properties of vanadium dioxide and vanadium pentoxide thin films. Appl. Opt. 1991, 30, 2782-2787. [CrossRef]

7. Markov, P.; Marvel, R.E.; Conley, H.J.; Miller, K.J.; Haglund, R.F., Jr.; Weiss, S.M. Optically monitored electrical switching in $\mathrm{VO}_{2}$. ACS Photonics 2015, 2, 1175-1182. [CrossRef]

8. Kim, B.J.; Lee, Y.W.; Chae, B.G.; Yun, S.J.; Oh, S.Y.; Kim, H.T.; Lim, Y.S. Temperature dependence of the first-order metal-insulator transition in $\mathrm{VO}_{2}$ and programmable critical temperature sensor. Appl. Phys. Lett. 2007, 90, 023515. [CrossRef]

9. Strelcov, E.; Lilach, Y.; Kolmakov, A. Gas sensor based on metal-insulator transition in $\mathrm{VO}_{2}$ nanowire thermistor. Nano Lett. 2009, 9, 2322-2326. [CrossRef]

10. Lee, Y.W.; Kim, B.J.; Lim, J.W.; Yun, S.J.; Choi, S.; Chae, B.G.; Kim, G.; Kim, H.T. Metal-insulator transition-induced electrical oscillation in vanadium dioxide thin film. Appl. Phys. Lett. 2008, 92, 162903. [CrossRef]

11. Ruzmetov, D.; Gopalakrishnan, G.; Deng, J.; Narayanamurti, V.; Ramanathan, S. Electrical triggering of metal-insulator transition in nanoscale vanadium oxide junctions. J. Appl. Phys. 2009, 106, 083702. [CrossRef]

12. Ruzmetov, D.; Gopalakrishnan, G.; Ko, C.; Narayanamurti, V.; Ramanathan, S. Three-terminal field effect devices utilizing thin film vanadium oxide as the channel layer. J. Appl. Phys. 2010, 107, 114516. [CrossRef]

13. Driscoll, T.; Kim, H.T.; Chae, B.G.; Di Ventra, M.; Basov, D.N. Phase-transition driven memristive system. Appl. Phys. Lett. 2009, 95, 043503. [CrossRef]

14. Chen, Z.; Gao, Y.; Kang, L.; Du, J.; Zhang, Z.; Luo, H.; Miao, H.; Tan, G. VO-based double-layered films for smart windows: Optical design, all-solution preparation and improved properties. Sol. Energy Mater. Sol. C 2011, 95, 2677-2684. [CrossRef]

15. Stefanovich, G.; Pergament, A.; Stefanovich, D. Electrical switching and mott transition in $\mathrm{VO}_{2}$. J. Phys. Condens. Matter 2000, 12, 8837. [CrossRef]

16. Wu, B.; Zimmers, A.; Aubin, H.; Ghosh, R.; Liu, Y.; Lopez, R. Electric-field-driven phase transition in vanadium dioxide. Phys. Rev. 2011, 84, 241410. [CrossRef] 
17. You, Z.; Xiaonan, C.; Changhyun, K.; Zheng, Y.; Mouli, C.; Ramanathan, S. Voltage-triggered ultrafast phase transition in vanadium dioxide switches. IEEE Electron Device Lett. 2013, 34, 220-222.

18. Hu, B.; Ding, Y.; Chen, W.; Kulkarni, D.; Shen, Y.; Tsukruk, V.V.; Wang, Z.L. External-straininduced insulating phase transition in $\mathrm{VO}_{2}$ nanobeam and its application as flexible strain sensor. Adv. Mater. 2010, 22, 5134-5139. [CrossRef]

19. Pashkin, A.; Kübler, C.; Ehrke, H.; Lopez, R.; Halabica, A.; Haglund, R.F.; Huber, R.; Leitenstorfer, A. Ultrafast insulator-metal phase transition in $\mathrm{VO}_{2}$ studied by multi terahertz spectroscopy. Phys. Rev. B 2011, 83, 195120. [CrossRef]

20. Brockman, J.S.; Gao, L.; Hughes, B.; Rettner, C.T.; Samant, M.G.; Roche, K.P.; Parkin, S.S. Sub-nanosecond incubation times for electric-field-induced metallization of a correlated electron oxide. Nat. Nanotechnol. 2014, 9, 453. [CrossRef]

21. Chae, B.-G.; Kim, H.-T.; Youn, D.-H.; Kang, K.-Y. Abrupt metal-insulator transition observed in $\mathrm{VO}_{2}$ thin films induced by a switching voltage pulse. Physica B 2005, 369, 76-80. [CrossRef]

22. Gopalakrishnan, G.; Ruzmetov, D.; Ramanathan, S. On the triggering mechanism for the metal-insulator transition in thin film $\mathrm{VO}_{2}$ devices: Electric field versus thermal effects. J. Mater. Sci. 2009, 44, 5345-5353. [CrossRef]

23. Boriskov, P.P.; Velichko, A.A.; Pergament, A.L.; Stefanovich, G.B.; Stefanovich, D.G. The effect of electric field on metal-insulator phase transition in vanadium dioxide. Tech. Phys. Lett. 2002, 28, 406-408. [CrossRef]

24. Hormoz, S.; Ramanathan, S. Limits on vanadium oxide mottmetal-insulator transition field-effect transistors. Solid State Electron. 2010, 54, 654-659. [CrossRef]

25. Ko, C.; Ramanathan, S. Observation of electric field-assisted phase transition in thin film vanadium oxide in a metal-oxide-semiconductor device geometry. Appl. Phys. Lett. 2008, 93, 252101. [CrossRef]

26. Shao, Z.; Cao, X.; Luo, H.; Jin, P. Recent progress in the phase-transition mechanism and modulation of vanadium dioxide materials. NPG Asia Mater. 2018, 10, 581-605. [CrossRef]

27. Jian, J.; Wang, X.; Li, L.; Fan, M.; Zhang, W.; Huang, J.; Qi, Z.; Wang, H. Continuous tuning of phase transition temperature in $\mathrm{VO}_{2}$ thin films on c-cut sapphire substrates via strain variation. ACS Appl. Mater. Interfaces 2017, 9, 5319-5327. [CrossRef]

28. Hongwei, L.; Junpeng, L.; Minrui, Z.; Hai, T.S.; Haur, S.C.; Xinhai, Z.; Lin, K. Size effects on metal-insulator phase transition in individual vanadium dioxide nanowires. Opt. Express 2014, 22, 30748. [CrossRef]

29. Sang, J.; Wang, P.; Meng, Y.; Xu, X.; Sun, J.L.; Wang, Y.; Hua, Z.; Zheng, T.; Liu, Z.; Wang, C.; et al. Simple method preparation for ultrathin $\mathrm{VO}_{2}$ thin film and control: Nanoparticle morphology and optical transmittance. Jpn. J. Appl. Phys. 2019, 58, 050917. [CrossRef]

30. Schilbe, P. Raman scattering in $\mathrm{VO}_{2}$. Physica B 2002, 316, 600-602. [CrossRef]

31. Kim, H.T.; Chae, B.G.; Youn, D.H.; Maeng, S.L.; Kim, G.; Kang, K.Y.; Lim, Y.S. Mechanism and observation of motttransition in $\mathrm{VO}_{2}$-based two-and three-terminal devices. New J. Phys. 2004, 6, 52. [CrossRef]

32. Qazilbash, M.M.; Brehm, M.; Chae, B.G.; Ho, P.C.; Andreev, G.O.; Kim, B.J.; Yun, S.J.; Balatsky, A.V.; Maple, M.B.; Keilmann, F.; et al. Mott transition in $\mathrm{VO}_{2}$ revealed by infrared spectroscopy and nano-imaging. Science 2007, 318, 1750-1753. [CrossRef] [PubMed]

33. Srivastava, R.; Chase, L.L. Raman spectrum of semiconducting and metallic $\mathrm{VO}_{2}$. Phys. Rev. Lett. 1971, 27, 727. [CrossRef]

34. Vikhnin, V.S.; Goncharuk, I.N.; Davydov, V.Y.; Chudnovskii, F.A.; Shadrin, E.B. Raman spectra of the high-temperature phase of vanadium dioxide and model of structural transformations near the metal-semiconductor phase transition. Phys. Solid State 1995, 37, 1971-1978.

35. Sun, Y.; Jiang, S.; Bi, W.; Long, R.; Tan, X.; Wu, C.; Wei, S.; Xie, Y. New aspects of size-dependent metal-insulator transition in synthetic single-domain monoclinic vanadium dioxide nanocrystals. Nanoscale 2011, 3, 4394. [CrossRef] [PubMed]

(C) 2020 by the authors. Licensee MDPI, Basel, Switzerland. This article is an open access article distributed under the terms and conditions of the Creative Commons Attribution (CC BY) license (http://creativecommons.org/licenses/by/4.0/). 